\title{
Immune thrombocytopenia following COVID-19 mRNA vaccine: casuality or causality?
}

\author{
Federico Pasin ${ }^{1}\left[\right.$. Alberto Calabrese ${ }^{2} \cdot$ Laura Pelagatti $^{1}$
}

Received: 5 May 2021 / Accepted: 24 May 2021 / Published online: 7 June 2021

(c) Società Italiana di Medicina Interna (SIMI) 2021

Keywords COVID-19 $\cdot$ Immune thrombocytopenic purpura $\cdot$ Thrombocytopenia $\cdot$ Vaccine $\cdot$ mRNA

Dear Editor,

Cases of apparent secondary immune thrombocytopenia (ITP) after SARS-CoV-2 vaccination with both the Pfizer/ BioNTech and Moderna mRNA-vaccine versions have been recently reported and reached public attention with general alarm related with risks of serious and potentially lifethreatening consequences. [1-3]. It is still unclear whether this relationship between coronavirus disease-19 (COVID19) vaccination and thrombocytopenia is coincidental or causal. A wide array of aberrations may occur in the immune response during the ITP pathogenetic process. These may affect both the humoral immunity and cell-mediated immunity. The mechanism of post-vaccination thrombocytopenia is presumed to be immune-mediated and may be similarly related to hyperfunction of B cells observed in ITP. The signalling pathway of cytokine leads to a specific response from $\mathrm{CD} 4+\mathrm{T}$ cells specifically activated against modified glycoprotein (GP)IIb-IIIa on activated platelets. GPIIb-IIIa and/or GPIb-IX autoantigens were the most likely identified ligands in ITP for antiplatelet autoantibodies using antigenspecific assays. Favorable response is noted in most of the patients treated with corticosteroids and intravenous immunoglobulin (IVIG).

It is important to inquire about recent infections, medications, and vaccinations when assessing a patient presenting with signs and symptoms suggestive of ITP. Physicians should be aware of the occurrence of vaccine-induced ITP in patients who manifest a new onset of bleeding.

Federico Pasin

federico.pasin@ASST-cremona.it

1 Internal Medicine Unit, ASST Cremona, Oglio-Po Hospital, Viale Concordia 1, 26100 Cremona, Italy

2 Intensive Care Unit, AOUPR, Parma, Italy
Herein we report a case of immune thrombocytopenia following the initial dose of the Pfizer/BioNTech mRNA SARS-CoV-2 vaccine in a 84-year-old man. On day five post-vaccination, he experienced widespread petechiae (Fig. 1), especially on lower extremities, gum bleeding, bruising of the limbs, which prompted his presentation. Laboratory tests revealed severe thrombocytopenia with platelet count of $3 \times 10^{9} / \mathrm{L}$, normal white cell count, mild chronic anemia (hemoglobin: $10.6 \mathrm{~g} / \mathrm{dl}$ ), and normal coagulation test. One month prior to vaccination, the patient had a pletelet count of $220 \times 10^{9} / \mathrm{L}$. The patient had no personal and family history of bleeding or autoimmune disease. His medical history included essential tremor, localized bladder cancer, mild chronic renal failure and paroxysmal atrial fibrillation treated with apixaban at lower dose ( $2.5 \mathrm{mg}$ bid). Vital signs and the remainder of his exam were normal. He tested negative for hepatitis B, hepatitis C antibody, HIV, Epstein-Barr virus and cytomegalovirus serology. Immunologic studies (rheumatoid factor, ds-DNA and cyclic citrullinated peptide antibodies) were also negative. The nasopharingeal swab for SARS-CoV-2 was repeatedly negative. Additionally, SARS-CoV-2 IgG antibody testing was performed to rule out that a previous COVID-19 infection elicited the ITP experienced, however, SARS-CoV-2 antibody test was negative. Finally, the anti-platelet antibodies (GPIIb-IIIa) were positive while the anti-PF4-antibodies were negative. Apixaban was discontinued on the first day on admission. Other drugs such as dutasteride, primidone and promazine were continued because the patient had been following this therapy for a long time. The patient initially received one unit of platelet transfusion due to bleeding tendency and oral prednisone $(1 \mathrm{mg} / \mathrm{Kg} / \mathrm{day})$. Then, due to persistent severe thrombocytopenia, the patient received five daily doses of intravenous immunoglobulin $(400 \mathrm{mg} /$ $\mathrm{Kg}$ /day. On day 3 after admission and on day 8 after vaccination petechiae and oral bleeding decreased. On day 20 


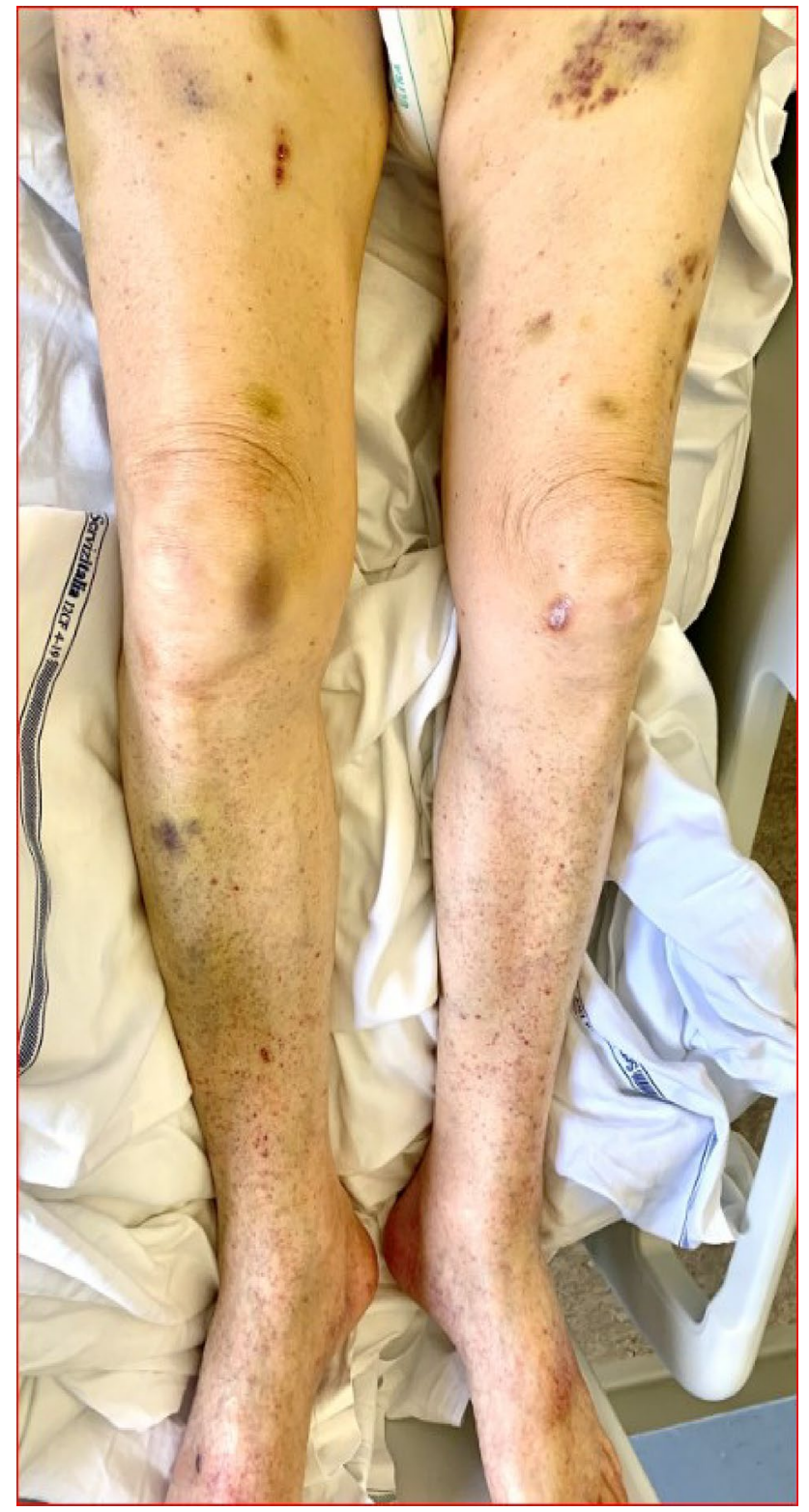

Fig. 1 Purpuric lesions on the patient's lower extremity, widespread petechiae and bruising on both legs

after admission, the platelets count began to rise to values of $63 \times 10^{9} /$ L (Fig. 2). The temporal relationship of the patient's presentation, 5 days post-vaccine administration suggests, but does not prove, the vaccine may be linked to
ITP development. Of note, there is also an increased risk of ITP after administration of vaccines like influenza, measles-mupms-rubella (MMR), hepatitis B, human papilloma virus, varicella and diphtheria-tetanus-pertussis (DPT) vaccines in children and adolescents [4].

Even in view of the uncertain relationship between SARS-CoV-2 vaccination and secondary ITP, it is worth considering possible mechanism by which this might occur. The vaccines-autoimmunity interplay is very similar to the established association between infections and autoimmunity. Infectious agents can cause or trigger autoimmunity through several mechanisms such as molecular mimicry, polyclonal activation, bystander activation and the presence of superantigens. Vaccines, as well as infections, activate immune-mediated mechanisms that can induce protective immunity. Thrombocytopenia has been reported after treatment with some anti-sense oligonucleotides. The epitope integrated within the vaccine antigen shares a similar structure with a self-antigen, driving towards self-reactivity. Furthermore, when polyclonal activation of B cells occurs, the increased $\mathrm{B}$ cell proliferation, antibody production and formation of circulating immune complexes may result in damage to self-tissues. Activation of antibodies and T cells responsible for the clearance of virus antigens may crossreact with antigens present on platelet membrane. The antibody-coated platelets are cleared by tissue macrophages, resulting in a shortened half-life of platelets. Notably, the increased risk of autoimmunity among recipients of a certain vaccine may stem not only from its antigen-mediated responses but also from other constituents of the vaccine, such as yeast proteins or extracts (no less than 5\% as residual traces from the manufacturing process), adjuvants and preservatives used to enhance its immunogenicity $[4,5]$.

We cannot exclude the possibility that the SARS-CoV-2 mRNA-vaccine have the potential to trigger de novo ITP, albeit rarely. Distinguishing vaccine-induced ITP from coincidental ITP presenting soon after vaccination is challenging at this time. Additional surveillance is needed to determine the true incidence of thrombocytopenia post COVID-19 vaccination. Further investigation is imperative to explicate the pathological mechanism, epidemiology, clinical manifestation, and treatment outcomes.

The case reported should not to be seen as a reason to avoid vaccination but, on the contrary, as an opportunity to carefully monitor and better know possible side effects and ways to mitigate possible complications. 
Fig. 2 Post-vaccination platelet count
VVaccination, $\mathbf{Z Z}$ Prednisone $1 \mathrm{mg} / \mathrm{kg} / \mathrm{die}, \bigcirc$ Platelet trasfusion, $\diamond \mathrm{IVIG} 400 \mathrm{mg} / \mathrm{kg}$

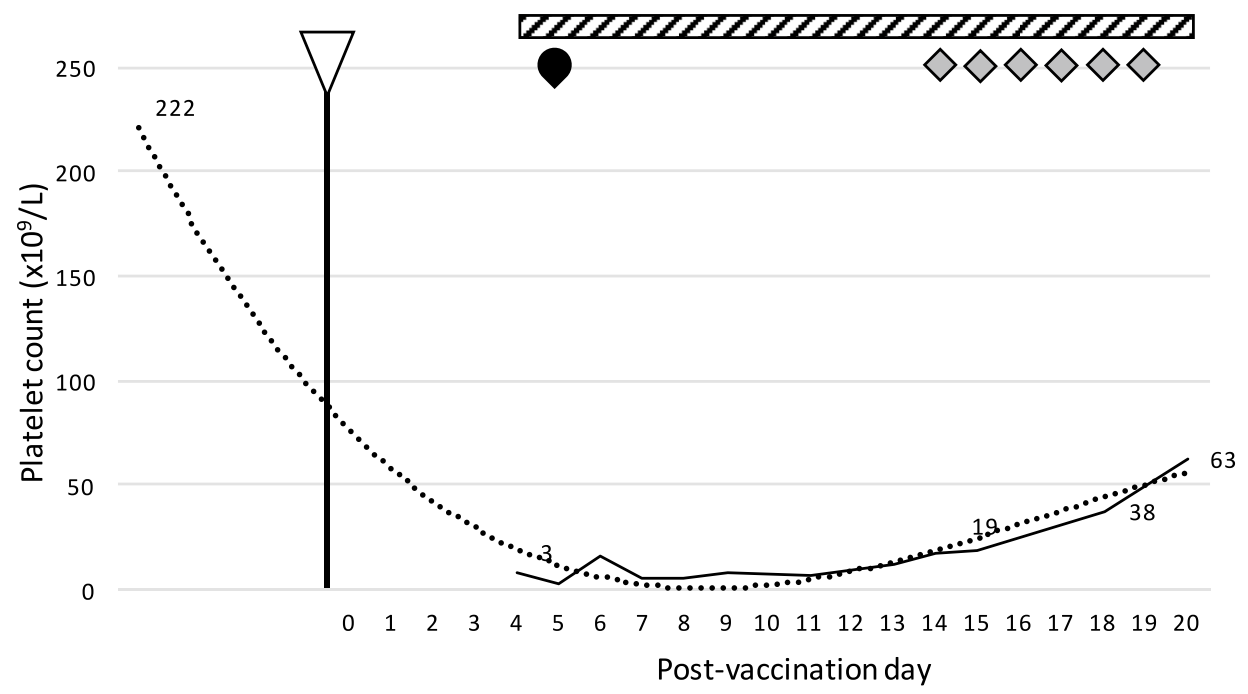

\section{Declarations}

Conflict of interest The authors declare no conflict of interest.

Human and animal rights statement This article does not contain any studies with human or animal subjects performed by the any of the authors.

Informed consent Informed written consent for publication of their details was obtained from the patient.

\section{References}

1. Tarawneh $\mathrm{OH}$, Tarawneh HS (2021) Immune thrombocytopenia in a 22 -year-old post Covid-19 vaccine. Am J Hematol. https:// doi.org/10.1002/ajh.26106
2. Helms JM, Ansteatt KT, Roberts JC, Kamatam S, Foong KS, Labayog JS, Tarantino MD (2021) Severe, refractory immune thrombocytopenia occurring after SARS-CoV-2 vaccine. J Blood Med 6(12):221-224

3. Lee EJ, Cines DB, Gernsheimer T, Kessler C, Michel M, Tarantino MD, Semple JW, Arnold DM, Godeau B, Lambert MP, Bussel JB (2021) Thrombocytopenia following Pfizer and Moderna SARS-CoV-2 vaccination. Am J Hematol 96(5):534-537. https:// doi.org/10.1002/ajh.26132 (Epub 2021 Mar 9)

4. Perricone C, Ceccarelli F, Nesher G et al (2014) Immune thrombocytopenic purpura (ITP) associated with vaccinations: a review of reported cases. Immunol Res 60:226-235

5. Rinaldi M, Perricone C, Ortega-Hernandez OD et al (2014) Immune thrombocytopaenic purpura: an autoimmune cross-link between infections and vaccines. Lupus 23:554-567

Publisher's Note Springer Nature remains neutral with regard to jurisdictional claims in published maps and institutional affiliations. 